\title{
Physics as Information Processing ${ }^{1}$
}

\author{
Giacomo Mauro D’Ariano \\ QUIT Group, Dipartimento di Fisica “A. Volta”, 27100 Pavia, Italy, http://www.qubit.it \\ Istituto Nazionale di Fisica Nucleare, Gruppo IV, Sezione di Pavia
}

\begin{abstract}
I review some recent advances in foundational research at Pavia QUIT group. The general idea is that there is only Quantum Theory without quantization rules, and the whole Physicsincluding space-time and relativity-is emergent from the quantum-information processing. And since Quantum Theory itself is axiomatized solely on informational principles, the whole Physics must be reformulated in information-theoretical terms: this is the It from Bit of J. A. Wheeler.

The review is divided into four parts: a) the informational axiomatization of Quantum Theory; b) how space-time and relativistic covariance emerge from quantum computation; c) what is the information-theoretical meaning of inertial mass and of $\hbar$, and how the quantum field emerges; d) an observational consequence of the new quantum field theory: a mass-dependent refraction index of vacuum. I will conclude with the research lines that will follow in the immediate future.
\end{abstract}

Keywords: Foundations of Physics, Axiomatics of Quantum Theory, Special Relativity, Quantum Field Theory

PACS: 03.65.-w

\section{INTRODUCTION}

After more than a century Quantum Theory (QT) has never been falsified in any experiment, and maintains its unprecedented predicting power. However, the derivation of its mathematical framework from practical principles has remained an open problem. Stimulated by the work of Lucien Hardy - who gave a first set of simple principles for QT [1] - and by Chris Fuchs_-who argued that QT is a special kind of information "and only a little more" [2] — in the summer of 2003 I started my axiomatization program. My dissatisfaction with Lucien's principles was that they were still of mathematical nature, whereas, as regards Chris, I couldn't imagine what was his little more. I presented the first very preliminary results of the program at the 2005 Vaxjo conference [3], introducing the operational axiomatization framework and providing a first list of candidate principles. In the recent article [4] I reached a stable set of purely operational postulates, though still incomplete. QT was there regarded as a set of rules that are sufficient for the experimenter to make predictions on future events on the basis of suitable tests. The postulates were: Causality, Local Observability, and the existence of Faithful States that allow calibrations of instruments and local preparation of joint states. Such postulates have shown an unprecedented power, excluding all known probabilistic theories except QT. The axiomatization program later continued in collaboration with my former students G. Chiribella and P. Perinotti. In Ref. [5] we have discovered the full potential of

\footnotetext{
${ }^{1}$ Work presented at the conference Advances in Quantum Theory held on 14-17 June 2010 at the Linnaeus University, Växjö, Sweden.
} 
a new Purifiability postulate, and started afresh with a new approach that provides a diagrammatic way of proving theorems. From the postulates we derived essentially all the relevant features of QT and Quantum Information, including dilation and error correction theorems, teleportation, no-cloning, and more. In the fall of 2009 we reached a complete set of postulates, that have been under scrutiny for the entire last year, and which seem to have finally reached the state of a manuscript $[6,7]$ (of the two references the former is intended to be a popularization, whereas the latter contains the precise formulation and the mathematical proofs). QT is derived from six principles that can be entirely formulated in the common language of computer programming: the original probabilistic operational approach is indeed nothing but the most general information-processing framework. We still don't know if the set of postulates is minimal (logical independence of axioms is knowingly an hard problem): indeed, as mentioned, just Causality, Local Discriminability and Purifiability are sufficient to derive all the relevant informational features of QT. Nevertheless, the new postulates are essential in the mathematical derivation. I will briefly review the postulates in Sect. 2. Let me mention here that among the Lucien's five reasonable axioms [1] Causality was completely overlooked: the postulate is indeed so natural that remained hidden in the framework. However, as shown in Refs. $[4,5]$, it is easy to build up a theory similar to QT without causality, and the relevance of recognizing Causality as a postulate is obvious in view of possible non-causal variations of QT for a theory of Quantum Gravity.

At the beginning of October 2009 I realized that the informational axiomatization program could go well beyond the narrow boundaries of QT and involve Relativity and Quantum Field Theory (QFT), i. e. the whole Physics. The point is: What is QFT more than just an infinite set of quantum systems locally interacting, i. e. a huge quantum computer? But then, Relativity and space-time itself should be emergent features of the quantum computation. In other words, Special Relativity must be derived from Causality.

The idea of deriving the geometry of space-time from a purely causal structure (such as a quantum computer) has been a long-term program initiated by Rafael Sorkin and collaborators [8] more than two decades ago! However, I wanted to see how the Lorentz transformations can be derived from causality. Remembering the TomonagaSchwinger foliation in their approach to QFT, I recognized the strict similarity with the foliation that Blute, Ivanov, and Panangaden [9] built up on causal networks (Lucien was perceiving the same point at the same time [10]). I whence realized that one should be able to see the Lorentz transformation on the quantum circuit by building up two different global foliations and comparing their synchronous sets of events. In this way I discovered that, upon introducing the metric as simple event-counting, from the causal network space and time emerge already endowed with the relativistic covariance. It was a startling experience to actually see the Lorentz time-dilation and length-contraction on the quantum circuit!

In writing the conference proceedings of my 2009 talk in Vaxjo [11] I couldn't resist reporting this idea (it will be briefly revisited here in Sect. 3). Five months after I posted the manuscript on the web, I was happily surprised by the appearance on the web of the work by Kevin Knuth and his student Newshaw Bahreyni [12], where they showed how the Minkowski signature can be derived from a causal poset. They indeed also claimed the derivation of the Lorentz transformations, but after careful reading their 
manuscript - indeed very interesting in consideration of the generality of their notion of "event"-I couldn't understand their derivation. In addition, I was unable to find the origin of what looked as a trivial error - the velocity at power four instead of two in the Lorentz transformation. In the meanwhile, with my student A. Tosini we worked out an analytical derivation of the Lorentz transformations [13] based on the idea of Ref. [11], using a topological homogeneous causal network. Later I asked Kevin about the apparent mistake in his manuscript, and it turned out that its fixing needed a change of the whole derivation - the good news was that, as a byproduct, the new derivation seemed to require the notion of spin! I still have not analyzed the second version of Kevin's manuscript (I'm still waiting for a self-contained forthcoming version), but in my opinion there is a crucial ingredient in the derivation of the Lorentz transformations-which still I don't understand how Kevin can avoid-i. e. the assumption of uniform topology of the causal network, corresponding to the Galileo principle: we will come back to this point later. But, let me say: everything looked so damned interesting! Later I discovered other related very interesting works by other authors in much earlier literature, such as Stephen Wolfram's idea that the Lorentz transformations might be derived from a cellular automaton evolution [14], Leslie Lamport's work [15] on the problem of time-ordering of events in distributed systems, and, dulcis in fundo, a very interesting derivation of the Lorentz composition of velocities based on a simple random-walk in the book of Irving Stein[16]—who, at the antipodes of the informational approach, seeking an ontology for Physics concludes that it is non classical!

Thus, after years of research spent in the tunnel of pure quantum theory, I finally saw the outdoor light of "space" and "time", and experienced the joy of entering the territory of Relativity and QFT. Under enthusiasm, I immediately tried to see what could be said about QFT: the program of translating the whole Physics into informationtheoretic terms looked huge and very ambitious. The first question that I was able to address was: what is the informational meaning of mass? The answer was simple: the Dirac's Zitterbewegung. Indeed, the inertial mass is nothing but the slowing-down of the information flow due to repeated changes of direction. In this way one can define mass in terms of the coupling between left and right-propagating information flows, which is expressed in terms of the Compton wavelength. The statement of the equivalence of this notion of mass with the usual notion (in the conventional units) leads also to recover $\hbar$ simply as the conversion factor between the two! I will briefly report about this point in Sect. 4. I liked this very much, since I always wanted to make Quantum Mechanics independent from $\hbar$, the notion of particle, and the quantization rule. Why? For many reasons. First: I never liked the quantization rules: why we should start from the theory of Classical Mechanics - that we know is incorrect - to derive QT-which we know is correct? Second: the notion of particle as an ontology becomes inconsistent with the wave-particle dualism. Third: the notion of particle becomes totally evanescent as an ontology in QFT, where it is just a quantum state - a state of the field! I wanted to have the three things- $\hbar$, notion of particle, and quantization rules-as emergent.

Again under the enthusiasm for the last findings, I immediately tried to work-out a set of toy models for the new Computational QFT, and rushed to write them on the same Vaxjo proceedings[11]. The result was that I made my own mistake: in order to make calculations easy I considered a quantum computer with gates infinitesimally close to the identity... without realizing that, in this fashion I couldn't derive Relativity 
anymore from the information-processing, since the maximal information speed must have been infinite! (I hope that the reader will forgive me: proceedings sometimes can be mistaken when they present work in progress). I later understood that, on the contrary, the new Quantum Computational Field Theory (QCFT) was very different from the usual quantum-computer simulation of Quantum Mechanics (see e. g. $[17,18]$ ), since it must have all unitary transformations very far from the identity, e. g. unitary swaps describing an information flow in a fixed direction at the maximum causal speed of 1 gate/1 step. QCFT must be something closer to the quantum cellular automaton of Ref. [19]. And in order to recover the continuum we should not take the limit of vanishing $a$ and $\tau$, but take instead the thermodynamic limit of infinitely many gates.

One of the good news about the new QCFT, is that it has observational consequences. For example, the request of unitariety for an evolution linear in the field leads to a massdependent vacuum refraction index. As I will show in Sect. 5, this phenomenon is simply a consequence of the normalization of the rows of the unitary matrix giving the linear evolution of the field: if we increase the mass (i. e. the coupling between modes), then we need to decrease the maximal propagation speed. This is a general feature of QCFT, and is due to the fact that QCFT respects causality, namely no information can propagate at a speed greater than $c$.

\section{QUANTUM THEORY AS INFORMATION THEORY}

In this section I will briefly review the six informational principles for QT of Ref. [6]. As mentioned, the principles can be formulated in the common language of computer programming. We just need to add few additional specifications. We call information processing the equivalence class of subroutines that achieve the same input-output relation. The subroutine corresponds to what it is usually called event in the language of operational probabilistic theories of Refs. [4, 5], whereas the equivalence class of the information processing corresponds to the notion of transformation. These are represented in form of a box with wires as follows

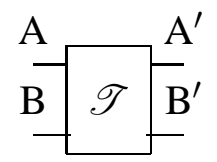

where the left/right wires represent input/output registers on which information is $\mathrm{read} / \mathrm{written}$, and different letters denote different types of register. The register corresponds to the notion of system in the operational framework. We can compose processings connecting input with outputs of the same type as follows

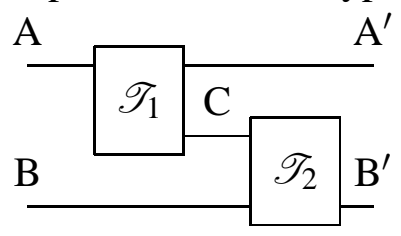

We should keep in mind that the circuit representation is not a flow-diagram, but represents the entire run (i. e. if we send the output to the input of a previously called processing we will not draw a loop, but instead we will redraw the same box twice). Therefore, 
precisely a box represents a single call of the processing. The entire processing is thus described by a DAG (directed acyclic graph). For subroutines and processings - as for events and transformations-it is possible to perform both coarse-graining and refinement, e. g. a subroutine can be divided into alternative subroutines whose occurrence generally depends on the input (for example, the "factorial" we can be divided into two alternatives $-\mathrm{n}=0$ and $\mathrm{n}>0-$ using the subroutine "return 1 " for $n=0$ and the subroutine "return $n * f(n-1)$ " for $n>0)$. The complete set of alternative processings corresponds to the operational notion of test (collection of events), and is represented by a single box as follows

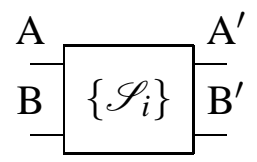

We name the set of all possible constituents of a processing its refinement set, and call indivisible a processing with trivial refinement set. The data-input and data-output are themselves information processings: the initialization and readout, respectively, corresponding to the operational notions of state and effect. These are represented as follows
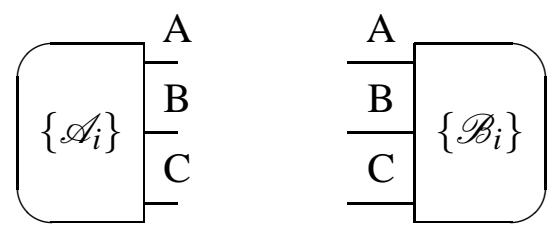

Thus an "indivisible initialization" is just the same as a pure state. An initialization followed by a processing can itself be regarded as a different initialization, namely

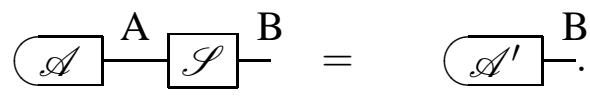

Finally, the domain of a processing is the set of its possible initializations, its range is the set of its possible readouts. An initialization is specific when its refinement set is not the whole set of initializations (in the operational language this means that the state is on the boundary of the convex set). Two initializations $\mathscr{A}_{1}$ and $\mathscr{A}_{2}$ are discriminable when there exists a readout that occurs with different probabilities on the two initializations, whereas the discrimination is perfect when the two probabilities are zero and one.

We are now ready to understand the postulates:

P1 Causality: The occurrence probability of a component processing is independent on the choice of the processing at its output (i. e. information flows from input to output, and not viceversa).

P2 Local Readability: We can discriminate two initializations of multiple registers by readouts on single registers.

P3 Reversibility and Indivisibility of Computation: Every information processing can be achieved through a reversible one, by adding auxiliary input registers in such a way that the joint initialization is indivisible.

P4 Indivisibility of Processing Composition: The processing corresponding to the input-output sequence of two indivisible processings is itself indivisible.

P5 Discriminability of Specific Initializations: For any specific initialization there exists another initialization that can be perfectly discriminated from it. 
P6 Lossless Compressibility: For any initialization there exists an encoding which is perfectly decodeable on its refinement set, and the encoded initialization is not specific.

The six postulates involve different aspects of the information processing, and each one generates a range of logical consequences that would require a long discussion: the reader is addressed to Refs. [6,7] for details. Here I just mention that Postulates P1, P2 and P4 correspond to Causality, Local-Discriminability, and Atomicity of Evolution of Ref. [4], respectively. P3 is the purification postulate of Ref. [5]. Postulates P5 and P6 are new. I already briefly commented on P1 in the introduction. Regarding the other postulates I just want to remind that P2 (Local Discriminability) is the origin of the complex tensor product and reconciles holism with reductionism. P3 synthesizes the parallelism and reversibility of quantum computation, and is the most "quantum" postulate, in the sense that all postulates apart from P3 are satisfied by classical theory (P3 is not satisfied by PR boxes [20]). P4 may look obvious: however, there is no reason why the same processing obtained by composing two indivisible ones could not be itself achieved in principle by a subroutine which is divisible. P5 and P6 also seem obvious, but it is easy to construct a theory that violates P5, whereas P6 becomes non trivial in a general information-processing framework with different types of registers. P6 is the starting point of Shannon's and Schumacher's compression. I want to emphasize that currently no known theory satisfies P1, P2, and P3 apart from QT, and, as shown in [5], $\mathrm{P} 3$ is the basis of most quantum information protocols. However, in the derivation of QT in [7] P5 and P6 play an essential role. The reader is addressed to Refs. [6, 7] for a thorough illustration of postulates and for the mathematical derivation of QT from them.

\section{EMERGENCE OF SPACE-TIME AND SPECIAL RELATIVITY FROM INFORMATION PROCESSING}

In Ref. [11] I showed how Special Relativity emerges from the local causality of a computational network. The basic idea is to make metric emerge from pure eventcounting from the purely topological structure of the computation. The wires in the quantum circuit represent causal connections, whence they can be stretched without affecting the information processing. Fig. 1 (from Ref. [11]) illustrates a factor-two Lorentz time-dilation and space-contraction. The two reference-systems correspond to the usual horizontal foliation and to the oblique staircase foliation drawn in the first circuit, respectively. Upon stretching the wires in order to put the synchronous slices horizontally - so that the vertical axis represents time-we obtain the second circuit, which is topologically identical to the first one. Time-dilation and space-contraction emerge as a consequence of a resulting reduced density of independent events, whereas time-dilation comes from a larger number of events during the same tic-tac of an Einstein clock (light bouncing between two mirrors). With Alessandro Tosini we gave an analytical derivation of the Lorentz transformations from a topologically uniform causal network [13]. Topological uniformity plays the role of the Galileo principle, i. e. the invariance of the physical law with the reference system - the physical law being the causal connection-rule of the network, i. e. a repeated tile of the pattern. Within a 

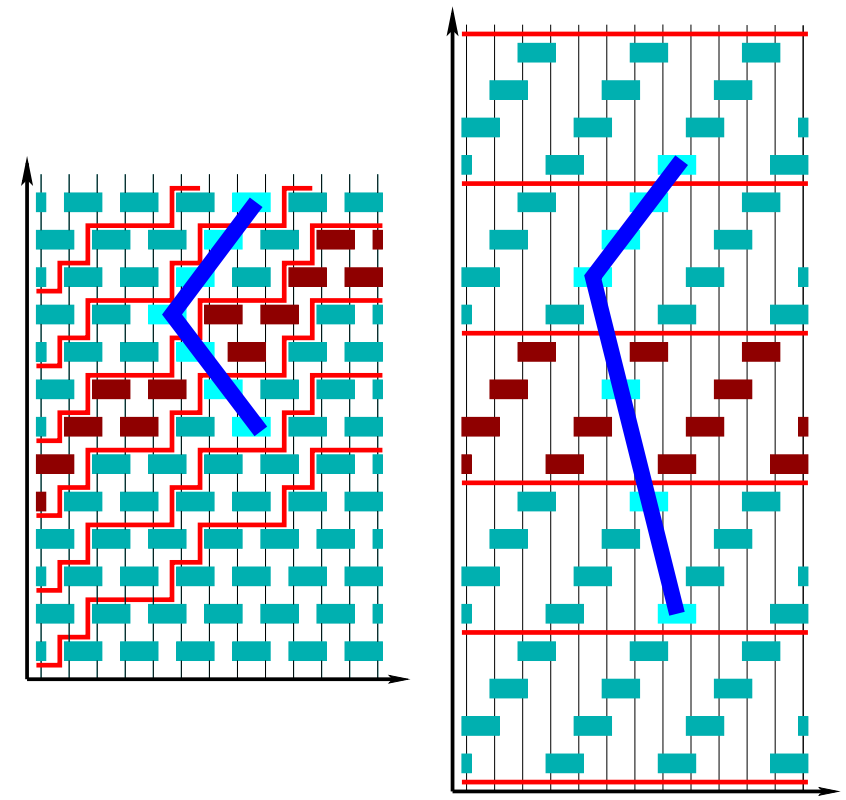

FIGURE 1. Illustration on how Special Relativity emerges from Local Causality. A chain of events representing a clock tic-tac (light bouncing between two mirrors) is highlighted in a topologically uniform computational circuit (on the left). On the same circuit a global uniform foliation is represented as a set of thin staircase lines. The second circuit is obtained from the first one upon stretching wires in order to put the synchronous slices horizontally, so that the vertical axis represents time. The corresponding clock tic-tac is highlighted. The Lorentz space-contraction emerges as a consequence of the reduced density of events: the distance between mirrors changes from two to one events. Time-dilation is evident by counting the number of events during the complete tic-tac: eight on the left, sixteen on the right.

single frame the Galileo principle is just uniformity of space and time: however, the causal network captures the conventionality of homogeneity of space and time-a long debated problem in Special Relativity (see e. g. books [21, 22])—while retaining the Galileo principle. In a homogeneous causal network it is easy to see how causality is sufficient to guarantee a maximum speed of information flow as one-event per step, corresponding to a line at $45^{\circ}$ in Fig. 1. Simultaneity in relation to an observer is introduced as follows. The observer is just an unbounded causal chain. Thanks to the topological homogeneity, we can translate the observer to any event in the network: simultaneity is defined by fixing an origin on the observer chain, placing the origin of two identical translated observers over the two events, sending light-signals from these events toward the other observer, and then comparing the number of clock tic-tacs for each observer (for details see Ref. [13]). Depending on the shape of the observer chain, one may have situations in which there are no synchronous events. However, for periodic observers (each ziz-zag is a clock tic-tac) there always exist infinitely many simultaneous events. The notion of simultaneity allows us to associate each observer with a foliation of the causal network, and using the foliation on can define coordinates, by which the Lorentz transformations are then derived. It is worth re-emphasizing that the whole procedure for defining space and time coordinates is made only by event-counting on the causal network. An essential ingredient in the transformation is a coarse-graining of 
events, corresponding to the usual rescaling of Minkowski space to restore symmetry between the observers (this coarse-graining is also related to the event-sparseness issue raised in Ref. [24] for Lorentz-transformed regular lattices of points).

\section{THE MEANING OF THE INERTIAL MASS AND OF $\hbar$}

2 As already said in the introduction, the information-theoretic meaning of inertial mass is simply the slowing-down of the information flow due to repeated changes of direction, namely the Dirac's Zitterbewegung. Thus, the inertial mass is the coupling between left and right propagating flows of information. A coupling between left/and right propagating flows producing a periodic oscillation of direction is described by the differential equation

$$
\widehat{\partial}_{t}\left[\begin{array}{l}
\phi^{+} \\
\phi^{-}
\end{array}\right]=\left[\begin{array}{cc}
v \widehat{\partial}_{x} & -i \omega \\
-i \omega & -v \widehat{\partial}_{x}
\end{array}\right]\left[\begin{array}{l}
\phi^{+} \\
\phi^{-}
\end{array}\right]
$$

where $\phi^{ \pm}$denote the operators for the left/right propagating flows, $v$ is the speed of the flow over the network, and $\widehat{\partial}_{x}$ and $\widehat{\partial}_{t}$ are finite-difference derivatives. The coupling constant is denoted by the oscillation angular frequency $\omega$. Eq. (1) has the same form of a Dirac equation (without spin), which means that the quantum-information processing simulates a Dirac field. This leads us to write the coupling constant in terms of the Compton wavelength $\lambda$ as $\omega=c \lambda^{-1}$, and this allows us to establish the following relation between the mass $m$ expressed in usual units (grams) and the informational mass $\omega$

$$
m=\frac{\tau^{2}}{a^{2}} \hbar \omega=\frac{1}{c^{2}} \hbar \omega .
$$

In Eq. (2) $a$ and $\tau$ denote the topon and the chronon, respectively-i. e. the conversion factors from pure event-counting to the usual units of space and time: in other words, they are the minimum space and time distances between gates in the informationprocessing. Eq. (2) also provides a meaning for the Planck constant: $\hbar$ is the conversion factor in the equivalence between the informational notion in $\mathrm{sec}^{-1}$ and the customary notion in $\mathrm{Kg}$ of the inertial mass. It is worth noticing that Eq. (2) also corresponds to the usual Planck formula in terms of particle rest energy, which are thus reinterpreted as the equivalence between the two notions of mass.

\section{THE MASS-DEPENDENT REFRACTION INDEX OF VACUUM}

As mentioned in the introduction, the new QCFT has observational consequences. For example, the request of unitariety and linearity in the field for the evolution lead to a mass-dependent vacuum refraction index. This is a general feature of QCFT, and does not depend on details of the quantum circuit. It is due to the fact that QCFT respects causality, namely no information can propagate at a speed greater than $c$, whereas in a

\footnotetext{
${ }^{2}$ For this section see also Ref. [23], which was written after the present manuscript was sent to press.
} 
customary simulation by a quantum computer with gates infinitesimally close to the identity, Lorentz covariance is recovered only in the continuum limit. I will now briefly outline the derivation of the mass-dependent refraction index of Ref. 5. In the same reference you can also find a detailed evaluation for a specific quantum circuit.

In the following we will use the notation $\phi(x)=\left[\phi^{+}(x), \phi^{-}(x)\right]^{T}$ for a generic spinless scalar field (either Boson or Fermion) in one space-dimension, $+(-)$ denoting the right- (left) propagating components. The time evolution of the field is given by $\phi(x, t)=U_{t} \phi(x) U_{t}^{\dagger}$, where $U_{t}$ is the global unitary evolution of the field up to time $t$, and $\phi(x):=\phi(x, 0)$. One has $|\phi(x, t)\rangle=\phi(x, t)^{\dagger}|0\rangle=U_{t}|\phi(x)\rangle=U_{t} \phi(x) U_{t}^{\dagger}|0\rangle$ and the vacuum must be left invariant, namely $U_{t}^{\dagger}|0\rangle=|0\rangle$.

The discretized version of the field is given by $\phi_{n}:=a^{\frac{1}{2}} \phi(n a)$, with $n \in \mathbb{Z}$. In QCFT there is no Hamiltonian: all gates produce finite transformations, and the unitary $U_{t}$ is the evolution due to all gates involved: in order to evaluate the evolution for few chronons of a single system - i. e. the field in a given location - one needs to consider only a finite set of gates. We can define an Hamiltonian in terms of a coarse-grained time-derivative of the field as follows

$$
\mathrm{H}_{\mathrm{gate}}^{(2 \mathrm{k})} z:=i \frac{z(k \tau)-z(-k \tau)}{2 k \tau}=: i \widehat{\partial}_{t} z
$$

where $z$ denotes any field component at any given position, and the caret on the partial derivative reminds us that it is discrete and coarse-grained. The field evolution is linear, whence we need to consider gates that transforms the fields linearly. We consider just a single space-dimension, but one can easily realize that the same reasoning apply to more dimensions. The coupling between left and right-propagating field components corresponds to the following Dirac-like equation (modulo unitarily equivalent representations)

$$
i \widehat{\partial}_{t}=c\left(i \zeta \sigma_{z} \widehat{\partial}_{x}+\lambda^{-1} \sigma_{x}\right)
$$

where we consider a general velocity $v=\zeta c$ and we write the coupling frequency in the convenient form $\omega=c \lambda^{-1}$. Using bipartite gates only, we need two rows of gates in the forward direction to get a term $\phi_{n+1}^{\varsigma}(\varsigma= \pm)$ in the unitary evolution of $\phi_{n}^{\varsigma}$, and similarly we need two rows of gates in the backward direction to get a term $\phi_{n-1}^{\varsigma}$, namely we need $k=2$ in Eq. (3). The general form of the circuit is depicted in Fig. 2. The

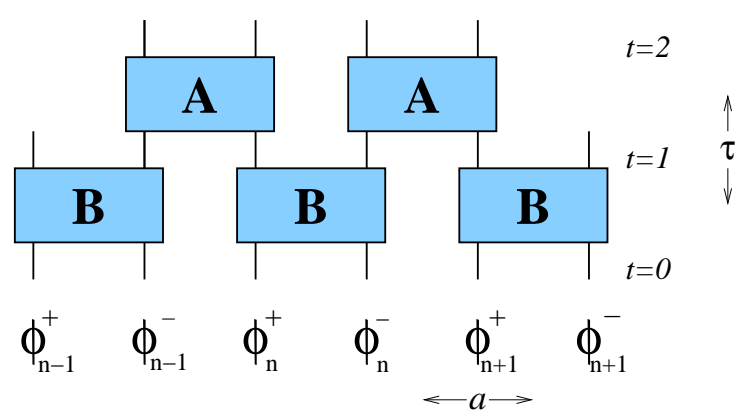

FIGURE 2. General quantum circuit made only of bipartite gates for a massive field equation. 
distance between the two adjacent positions $n$ and $n+1$ is $2 a$ (a right-propagating field covers it in two chronons), which means that the coarse-grained space derivative to be considered is $\widehat{\partial}_{x}=\frac{1}{4 a}\left(\delta_{+}-\delta_{-}\right)$. This means that in order to obtain Eq. (4) one must have the following overall backward and forward unitary interactions

$$
U_{f} \phi_{n}^{+} U_{f}-U_{b}^{\dagger} \phi_{n}^{+} U_{b}=\zeta\left(\phi_{n+1}^{+}-\phi_{n-1}^{+}\right)-4 i \frac{a}{\lambda} \phi_{n}^{-} .
$$

Therefore, since we consider gates involving only neighboring wires, the unitary evolutions must be of the form

$$
U_{f} \phi_{n}^{+} U_{f}^{\dagger}=\eta \phi_{n}^{+}+\zeta \phi_{n+1}^{+}+\gamma \phi_{n}^{-}, \quad U_{b}^{\dagger} \phi_{n}^{+} U_{b}=\eta \phi_{n}^{+}+\zeta \phi_{n-1}^{+}+\gamma^{\prime} \phi_{n}^{-},
$$

with $\zeta>0$ and $\gamma-\gamma^{\prime}=-4 i \frac{a}{\lambda}$. The two evolutions may include other terms that cancel each-other. But normalization of the row of the unitary matrix of the linear evolution corresponds to

$$
|\gamma|,\left|\gamma^{\prime}\right| \leqslant \sqrt{1-\zeta^{2}} \Longrightarrow \frac{2 a}{\lambda} \leqslant \sqrt{1-\zeta^{2}}
$$

which gives the lower bound for the vacuum refraction index

$$
\zeta^{-1} \geqslant \sqrt{1-\left(\frac{2 a}{\lambda}\right)^{2}}
$$

At the end of the section I want to comment about the realization of field (anti)commutations with local Pauli-matrix algebras, e. g. common qubits. The unitary transformation of each gate must correspond to a unitary linear combinations of the field operators of the input wires. These can be written in terms of local Pauli matrices using the Clifford algebraic construction

$$
\phi_{n}^{+}=\sigma_{n+}^{-} \prod_{k=-\infty}^{n-1} \sigma_{k+}^{z} \sigma_{k-}^{z}, \quad \phi_{n}^{-}=\sigma_{n-}^{-} \sigma_{n+}^{z} \prod_{k=-\infty}^{n-1} \sigma_{k+}^{z} \sigma_{k-}^{z} .
$$

Using the Fermionic Jordan-Schwinger realization of the unitary group $U$ (2) (of the unitary linear combinations of the fields operators of the two wires at the gate), it is easy to see the unitary transformation of gates are of the form

$$
A=A\left(\phi_{n}^{-}, \phi_{n+1}^{+}\right) \equiv A\left(\vec{\sigma}_{n-}, \vec{\sigma}_{(n+1)+}\right), \quad B=B\left(\phi_{n}^{+}, \phi_{n}^{-}\right) \equiv B\left(\vec{\sigma}_{n+}, \vec{\sigma}_{n-}\right),
$$

namely each gate is a function only of the qubit algebra of its wires. The possibility of generalizing the construction to more than one space-dimension, however, is still not obvious.

\section{CONCLUSIONS}

The new QCFT opens an extensive long-term research program. It represents the backbone of a complete translation of Physics into information-theoretic terms. In addition 
to needing only QT without quantization rules and giving Lorentz covariance for free, QCFT has the potential of solving many problems that plague QFT [11]—being naturally a lattice theory_-providing a new unified framework for particle physics.

The next immediate easy research steps will be to recover the commutation relations as emergent, to retrieve the unitary representation of the Lorentz group, to classify the vacuum states, to translate Lagrangian density into gates, to re-derive the Feynman's path integral. We need then to generalize the information processing to more than 1 space dimension. Thereafter, less easy problems need to be addressed, such as the issue of anticommuting fields (or parastatistics) and their realization with local operators in more than one space dimensions. Then we want to exploit the natural gauge invariance of the quantum circuit (local unitary transformations connecting the Hilbert spaces of different wires in a slice) with the aim of deriving a first prototype of gauge theory. Experimentally detectable effects will be the most interesting topic.

In the longer term we wants to explore how QCFT can help addressing the problem of Quantum Gravity. There are two possible main paths: either we adopt a strong version of the equivalence principle - in which the informational meaning of inertial mass coincides with the gravitational one-or we adopt a causal set approach to gravity. In the first case gravity should emerge as a quantum effect. In the second case we may need a non-causal variation of QT, e. g. by considering an information processing in a third-quantization fashion, where also the causal connections are treated as "states". A possible route is to consider super-gates as the switch-comb of Ref. [25].

Whichever the research process will be, QCFT is a very promising testing ground for foundations of QFT, an area of study that had never received the due attention, mostly due to the too technical character of QFT.

\section{Acknowledgments.}

I thank Seth Lloyd, Masanao Ozawa, Paolo Perinotti, Alessandro Tosini, Lucien Hardy, Rafael Sorkin, and Lee Smolin for very interesting discussions and suggestions. I'm grateful to researchers at the National Labs of Frascati and at Fermilab of Chicago for useful feedback and very encouraging support.

\section{REFERENCES}

1. L. Hardy, Quantum theory from five reasonable axioms, quant-ph/0101012v4 (2001).

2. C. A. Fuchs, Quantum Mechanics as Quantum Information (and only a little more), quant-ph/0205039 (2002).

3. G. M. D'Ariano, On the missing axiom of quantum mechanics, Quantum Theory, Reconsideration of Foundations - 3, Vâxjő, Sweden, 6-11 June 2005 (Melville, New York) (G. Adenier, A. Y. Khrennikov, and T. M. Nieuwenhuizen, eds.), (AIP, Melville, New York 2006) pp. 114.

4. G. M. D'Ariano, in Philosophy of quantum information and entanglement, A. Bokulich and G. Jaeger eds., (Cambridge University Press, Cambridge UK, 2010). Also arXive 0807.4383.

5. G. Chiribella, G. M. D’Ariano, P. Perinotti, Probabilistic Theories with Purification, Phys. Rev. A 81 062348 (2010).

6. G. Chiribella, G. M. D’Ariano, P. Perinotti, Quantum Theory is a Theory of Information (work in progress: see arXiv 2010-11) 
7. G. Chiribella, G. M. D'Ariano, P. Perinotti, Informational derivation of Quantum Theory arXiv 2011.6451 (2010).

8. L. Bombelli, J. H. Lee, D. Meyer, and R. Sorkin, Space-Time as a Causal Set, Phys. Rev. Lett. 59, 521 (1987).

9. R. Blute, I. Ivanov, and P. Panangaden, Discrete quantum causal dynamics, Int. J. Theor. Phys. 42 2025 (2003)

10. L. Hardy, Foliable Operational Structures for General Probabilistic Theories, arXiv: 0912.4740 (2009)

11. G. M. D'Ariano, On the "principle of the quantumness", the quantumness of Relativity, and the computational grand-unification, in CP1232 Quantum Theory: Reconsideration of Foundations, 5, edited by A. Y. Khrennikov (AIP, Melville, New York, 2010), pag 3. (Also arXiv:1001.1088)

12. K. H. Knuth, N. Bahreyni, arXiv: 1005.4172 (2010).

13. G. M. D'Ariano and A. Tosini, Space-time and special relativity from causal networks, arXiv: $1008.4805(2010)$

14. S. Wolfram. A New Kind of Science, Wolfram Media (Champaign, 2002).

15. L. Lamport, Time, clocks, and the ordering of events in a distributed system, Comm. ACM, 21558 (1978)

16. I. Stein, The Concept of Object As the Foundation of Physics, San Francisco State University Series in Philosophy, vol. 6 (Peter Lang Publishing, NY 1996)

17. I. Bialynicki-Birula, Weyl, Dirac, and Maxwell equations on a lattice as unitary cellular automata, Phys. Rev. D 496920 (1994)

18. B. M. Boghosian and W. Taylor, Simulating quantum mechanics on a quantum computer, Physica D: Nonlinear Phenomena 12030 (1998)

19. D.A. Meyer, From quantum cellular automata to quantum lattice gases, J. Stat. Phys. 85551 (1996)

20. G. M. D'Ariano and A. Tosini, Testing axioms for Quantum Theory on Probabilistic toy-theories, Quant. Inf. Proc. 9 95-141 (2010) (Special Issue on Foundations of Quantum Information) (also arXiv:0911.5409)

21. Harvey R. Brown, Physical Relativity: Space-Time Structure from a Dynamical Perspective (Clarendon Press, Oxford, 2005)

22. Max Jammer, Concepts of Simultaneity: From Antiquity to Einstein and Beyond (John Hopkins University, Baltimore Press 2006)

23. G. M. D'Ariano, The Quantum Field as a Quantum Computer, arXiv (2010)

24. F. Dowker, J. Henson, and R. D. Sorkin, Mod. Phys. Lett. A 191829 (2004).

25. G. Chiribella, G. M. D'Ariano, P. Perinotti, and B. Valiron, Beyond Quantum Computers, arXiv:0912.0195 